\title{
Effect of Radiation on the properties of ZnO Films and Substrates
}

\author{
A. M. Ibraheem \\ Department of Physics, College of Science, Sudan University of Science and Technology, Khartoum 11113, Sudan
}

\begin{abstract}
Zinc oxide (ZnO) films and substrates are important and attractive materials for applications in several fields. The Properties of $\mathrm{ZnO}$ films, glass and $\mathrm{ZnO} /$ glass have been studied by using ion beams. The effects of glass substrates on physical properties of ZnO films have been investigated. Details of calculations methods and results are given and discussed. The calculated values were compared with the published data. Good agreement between our results and published data is obtained.
\end{abstract}

Keywords: Zinc Oxide; Glass; SRIM; Physical Properties; Stopping power, PACS

\section{Introduction}

Zinc oxide $(\mathrm{ZnO})$ is one of the most intensely studied metal oxides. ZnO is a wide band gap oxide [1] and is a non-toxic material. It exhibits a large exciton binding energy [2] at room temperature. The $\mathrm{ZnO}$ thin films are promising materials and used in various applications $[3,4]$. There are different methods to prepare zinc oxide [5-11]. Up to now, a number of studies on $\mathrm{ZnO}$ films and various substrates including glass and flexible polymer have been conducted [12-14]. The substrate is very important for the growth of thin films in terms of the lattice and thermal mismatching between it and the film because it commonly leads to the development of stress in the deposited film. However, numerous parameters must be examined and evaluated in understanding the effects of radiation on polymer materials.

The present study investigates effects of proton beams on the physical properties of $\mathrm{ZnO}$ thin films on glass substrates. Calculations are underway to study the transport of ion beams in these films to get a better understanding of the effect of radiation. Radiation effects are predicted and compared on the basis of simulations of proton transport through layers of the of $\mathrm{ZnO}$ thin films on glass substrates.

\section{Materials and Methods}

\subsection{Materials details}

The samples ( $\mathrm{ZnO}$ and glass) were used to perform the calculations. The details of these samples are given in Table 1, 2 and 3. The samples were selected from SRIM code [15].

Table 1: ZnO Target description

\begin{tabular}{|c|c|c|c|c|}
\hline Element & Atomic Number & Weight $(\mathrm{amu})$ & Stoich & Atom\% \\
\hline Zinc, $\mathrm{Zn}$ & 30 & 65.39 & 1 & 50.33 \\
\hline Oxygen, O & 8 & 15.999 & 1 & 50.33 \\
\hline
\end{tabular}

Table 2: Glass (Soda Lima) Target description

\begin{tabular}{|c|c|c|c|c|}
\hline Element & Atomic Number & Weight (amu) & Stoich & Atom\% \\
\hline Oxygen, $\mathrm{O}$ & 8 & 15.99 & 60 & 60 \\
\hline Silicon, $\mathrm{Si}$ & 14 & 28.08 & 25 & 25 \\
\hline Sodium, $\mathrm{Na}$ & 11 & 22.99 & 10 & 10 \\
\hline Calcium, $\mathrm{Ca}$ & 20 & 40.08 & 3 & 3 \\
\hline Magnesium, $\mathrm{Mg}$ & 12 & 24.3 & 1 & 1 \\
\hline Aluminum, $\mathrm{Al}$ & 13 & 26.98 & 1 & 1 \\
\hline
\end{tabular}

Chemical structure unit of Glass (Soda Lima) is presented in Table 2. It contains 8 atoms of Oxygen, 14 of Silicon, 11 of Sodium, 20 of Calcium, 12 of Magnesium and 13 of Aluminum. Various physical and chemical methods based on different principles and processes are available for study $\mathrm{ZnO}$ and glass materials. It is well known that, when a material bombarded with energetic ions, many processes are initiated and this gives rise to a variety of physical and chemical phenomena.

Table 3: ZnO/Glass (Soda Lima) Target description

\begin{tabular}{|c|c|c|c|c|}
\hline Element & Atomic Number & Weight (amu) & Stoich & Atom\% \\
\hline Zinc, $\mathrm{Zn}$ & 30 & 65.39 & 50 & 25 \\
\hline Oxygen, $\mathrm{O}$ & 8 & 15.99 & 50 & 25 \\
\hline Oxygen, $\mathrm{O}$ & 8 & 15.99 & 60 & 30 \\
\hline Silicon, $\mathrm{Si}$ & 14 & 28.08 & 25 & 12.5 \\
\hline Sodium, $\mathrm{Na}$ & 11 & 22.99 & 10 & 5 \\
\hline Calcium, $\mathrm{Ca}$ & 20 & 40.08 & 3 & 1.5 \\
\hline Magnesium, $\mathrm{Mg}$ & 12 & 24.3 & 1 & 0.5 \\
\hline Aluminum, $\mathrm{Al}$ & 13 & 26.98 & 1 & 0.5 \\
\hline
\end{tabular}

\subsection{Methods details}

Computer simulation methods to calculate the motion of ions in a medium have been developed since the 1960s, and are now the dominant way of treating stopping power theoretically. The basic idea in them is to follow the movement of the ion in the medium by simulating the collisions with nuclei in the medium. The in this work, simulation was performed by Stopping and Range of Ions in Matter (SRIM). This program is used to calculate interaction of ions with matter. SRIM is a group of programs which calculate the stopping and range of ions (10eV-2GeV/amu) into matter. SRIM is using a quantum mechanical treatment of ion-atom collisions. A detailed description of the calculation method can be found in the Stopping and Range of Ions in Solid [15]. This code will accept complex targets made of compound materials with up to eight layers, each of different materials. It will calculate both the final 3D distribution of the ions and also all kinetic phenomena associated with the ion's energy loss. It can be used for physics of recoil cascades, physics of sputtering, the stopping of ions in compounds and stopping powers for ions in gases; this included radiation damage from neutron, electrons and photons. In SRIM program the 


\section{International Journal of Science and Research (IJSR) \\ ISSN (Online): 2319-7064 \\ Index Copernicus Value (2013): 6.14 | Impact Factor (2015): 6.391}

ion is perpendicular to the target surface, along the $\mathrm{x}$-axis at an angle defined as $0^{\circ}$. This angle may be changed to any angle from $0-89.9^{0}$. The change in ion direction is assumed to be in the XY plane. Normally, you select the default (99999) and interrupt SRIM when it has adequate statistics. Detailed descriptions and discussions of the physical background of these simulations can be found in literature [15]. To simulate the ion beam irradiation, a large number of ions are consecutively assumed to impinge on the target under a predefined angle and the transport of the ions through the target is simulated by SRIM. Because of $\mathrm{ZnO}$ and glass targets importance, we have used SRIM program for Stopping Power and Range calculations. In the SRIM codes, stopping power is defined as the energy required to slowing down the incident particle during its interaction with matter over a certain distance, and is mathematically expressed as [15]:

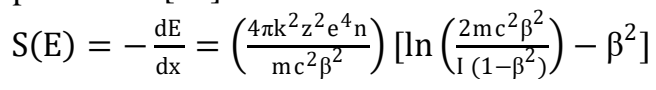

Where, $\mathrm{k}$ is $8.99 \times 109 \mathrm{~N} \cdot \mathrm{m}^{2} \cdot \mathrm{C}^{2}, z$ is atomic number, $e$ is charge of electron, $n$ is number of electron per unit volume of the target, $m$ is mass of electron at rest, $c$ is speed of light in vacuum, $\beta$ is a ratio of the speed of the incident particle to the speed of light, $I$ is an average excitation energy of the target. However, after losing energy due to electronic and nuclear interactions, the incident ion will eventually stop at a certain distance from the target surface and leave some vacancies in the target. The distance over which the ion totally stops is called the projected range $R(E)$, which satisfies [15]

$$
R=\int_{0}^{E_{0}}\left(-\frac{d E}{d x}\right) d E
$$

This quantity is known as the reciprocal stopping power range. Where, $\mathrm{E}_{0}$ is the incident ion energy at the target surface, $\mathrm{x}$ is the distance measured along the ion path, $\mathrm{dE} / \mathrm{dx}$ is the energy loss (which has both nuclear and electronic energy loss part) of the ion with energy E. The description of ions is given in Table 4.

Table 4: Ion description

\begin{tabular}{|c|c|c|c|c|}
\hline Element & Atomic & Mass & \multicolumn{2}{|c|}{ Ion Energy Range (keV) } \\
\cline { 4 - 5 } & Number & $(\mathrm{amu})$ & Lowest & Highest \\
\hline Hydrogen, H & 1 & 1.008 & 10 & 10000 \\
\hline
\end{tabular}

\section{Radiation Effects in Materials}

In this study Zinc oxide and Glass (soda -lime) substrates were selected and studied change of properties with proton treatment. Glass is a thermodynamically unstable state and has a defect structure compared to the crystal. Glass and its properties are subject to a variety of changes under the influence of high energy radiation. In general, effects extend from the reduction of specific ions to the collapse of the entire network. The effects of radiation on polymer materials are an area of rapidly increasing interest [16]. Polymers in particular are sensitive to radiation damage caused by X-ray, proton and electron irradiation [16]. Interaction of incoming particles with matter results in two major effects: ionization energy loss and nonionizing energy loss. Interactions of incoming particles which result in electronic excitation or ionization of atoms are referred to as ionization energy loss. In nonionizing energy loss processes, the energy imparted by the incident particles results in atomic displacements or in collisions where the primary knock-on atom remains in its lattice position, in which case the energy is converted to lattice vibrations (phonons). Displaced atoms can also undergo both electronic and displacement energy losses to dissipate their energy inside the medium. Secondary electrons created by ionization energy losses affect electrical properties of crystalline insulators in a transient manner, except if these electrons get trapped at electrically active point defects in the crystal lattice. Point defects that serve as charge-carrier traps or donors, arise in irradiated insulators as a result of atomic displacements, that is, of nonionizing energy loss. A heavy charged particle can transfer only a small fraction of its energy in a single electronic collision, and its deflection in the collision is negligible. It therefore has an almost straight trajectory in matter, losing energy continuously in small amounts through collisions with atomic electrons, leaving ionized and excited atoms along its path. For charged particles, such as protons, ionization, and electronic excitation, energy losses are represented by the electronic stopping power (also called collision stopping power, which is a misnomer, since all interactions can be considered collisions) of the material through which they propagate. Stopping power of a medium for a charged particle is the average linear rate of energy loss of the particle in the medium. It is, therefore, equal to the unrestricted linear energy transfer. Several semi-empirical stopping power formulas have been devised. Energetic heavy ion irradiations, on the other hand, produce energetic displacement cascades that can lead to direct formation of defect clusters within isolated displacement cascades, due to more energetic average recoil atom energies $(>10 \mathrm{keV})$. The units of nonionizing energy loss are typically $\mathrm{MeV} / \mathrm{cm}$, or $\mathrm{MeV} \mathrm{cm} 2 / \mathrm{g}$ if mass nonionizing energy loss is considered.

\section{Results and Discussion}

In this work, systematic calculations of the stopping powers and range for $0.01-10 \mathrm{MeV}$ protons in $\mathrm{ZnO}$, Glass and $\mathrm{ZnO} / \mathrm{Glass}$ targets are carried out using Ziegler's program SRIM 2013. Electronic stopping powers of $0.01-10 \mathrm{MeV}$ protons in $\mathrm{ZnO}$ target was calculated using SRIM to be in range $\left(0.02940-0.3036 \mathrm{MeV} / \mathrm{gmcm}^{2}\right)$, while nuclear stopping power was in range (0.0000147$0.002951 \mathrm{MeV} / \mathrm{gmcm}^{2}$ ). Figure 1 depicts the variation of electronic and nuclear stopping power of $0.01-10 \mathrm{MeV}$ protons with ion energy. For charged particles, such as protons, ionization, and electronic excitation, energy losses are represented by the electronic stopping power. 


\section{International Journal of Science and Research (IJSR) \\ ISSN (Online): 2319-7064}

Index Copernicus Value (2013): 6.14 | Impact Factor (2015): 6.391

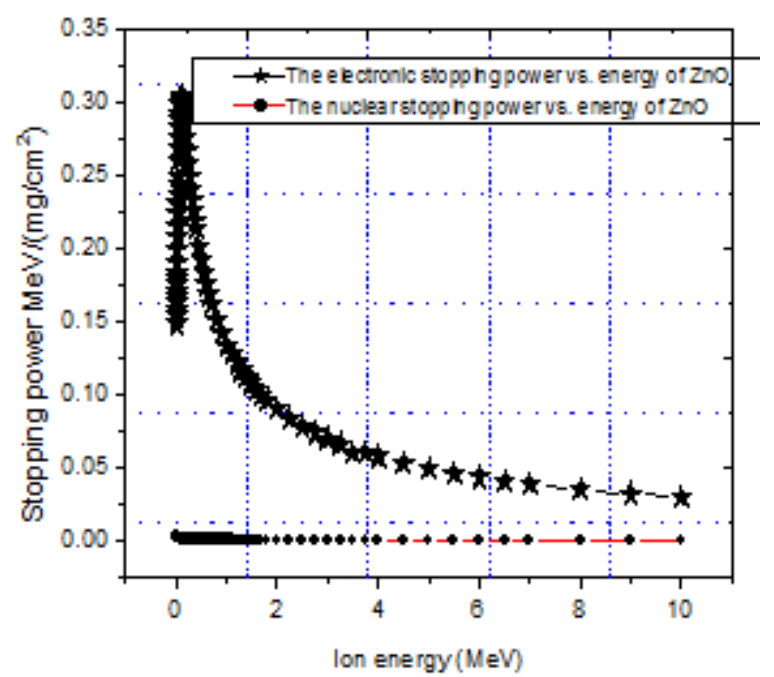

Figure 1: The stopping power of $\mathrm{ZnO}$ for protons, plotted versus ion energies

From this figure, it was clear that the electronic stopping power mechanism dominated the nuclear stopping power. The low electronic stopping powers of $10 \mathrm{MeV}$ protons in $\mathrm{ZnO}$ were $0.02940 \mathrm{MeV} /\left(\mathrm{mg} / \mathrm{cm}^{2}\right)$. While the high electronic stopping powers of $\mathrm{ZnO}$ were 0.3036 $\mathrm{MeV} /\left(\mathrm{mg} / \mathrm{cm}^{2}\right)$ at ion energy $0.110 \mathrm{MeV}$. The stopping power $\mathrm{S}$ increases as the particle velocity is decreased. The stopping number varies slowly with particle energy and is proportional to the atomic number $\mathrm{Z}$ of the absorber material.

Simulations of proton transport through thin layers of insulators were performed in the TRIM module of the SRIM software package [15]. Simulations used monoenergetic unidirectional beams, incident perpendicularly on the film's surface. Proton energies were varied from $10 \mathrm{keV}$ to $10 \mathrm{MeV}$. Each simulation run followed $10^{4}$ proton histories. Results in Figures 1 to 6 present the most illustrative examples of proton beam effects in the studied targets for the investigated proton energy range.

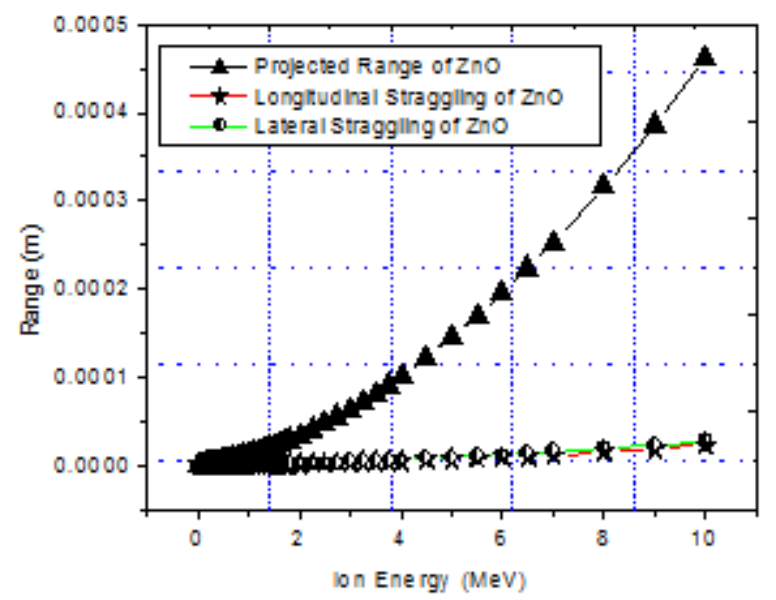

Fig. 2: The range of $\mathrm{ZnO}$ for protons, plotted versus ion energies

The projected range, the longitudinal range and the lateral range values of $0.01-10 \mathrm{MeV}$ protons in $\mathrm{ZnO}$ was calculated using SRIM to be $(0.0000001120-0.00046187 \mathrm{~m})$, $(0.0000000645-0.00002250 \mathrm{~m})$ and $(0.0000000555-$
$0.000028 .39 \mathrm{~m})$, respectively. Figure 2 depicts the variation of projected range, longitudinal range and lateral range of $0.01-10 \mathrm{MeV}$ protons with ion energy. The Figure 2 shows how the range of $\mathrm{ZnO}$ for protons increases until it reaches the maximum.

Electronic stopping powers of $(0.01-10 \mathrm{MeV})$ protons in glass target were calculated using SRIM to be $(0.03689$ $0.5519 \mathrm{MeV} / \mathrm{gmcm}^{2}$ ), while nuclear stopping power was $\left(0.00001832-0.004741 \mathrm{MeV} / \mathrm{gm} \mathrm{cm}^{2}\right)$. Figure 3 depicts the variation of electronic and nuclear stopping power of 0.01$10 \mathrm{MeV}$ protons with ion energy.

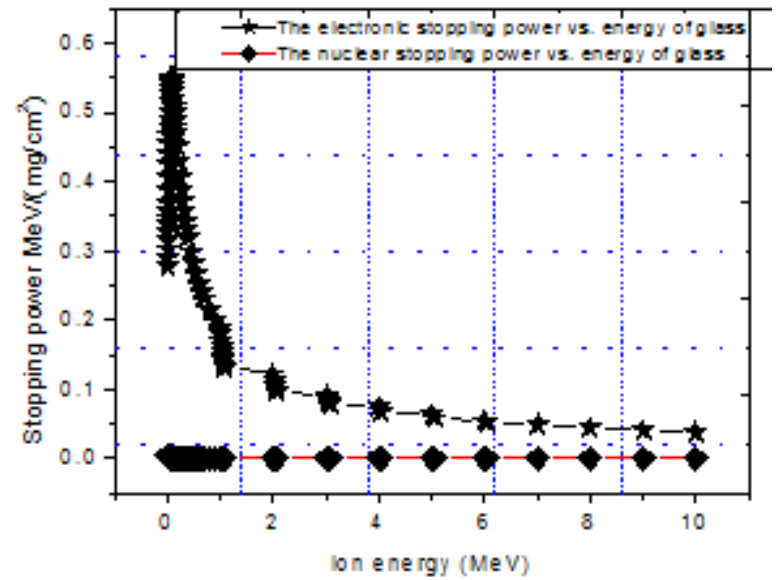

Figure 3: The stopping power of glass for protons, plotted versus ion energies

The projected range, the longitudinal range and the lateral range values of $0.01-10 \mathrm{MeV}$ protons in glass was calculated using SRIM to be $(0.0000001498-0.00066114 \mathrm{~m})$, $(0.00003010-0.0000000557 \mathrm{~m})$ and $(0.0000000533-$ $0.00002569 \mathrm{~m})$, respectively. Figure 4 depicts the variation of projected range, longitudinal range and lateral range of $0.01-10 \mathrm{MeV}$ protons with ion energy. The Figure 4 shows how the range of glass for protons increases until it reaches the maximum.

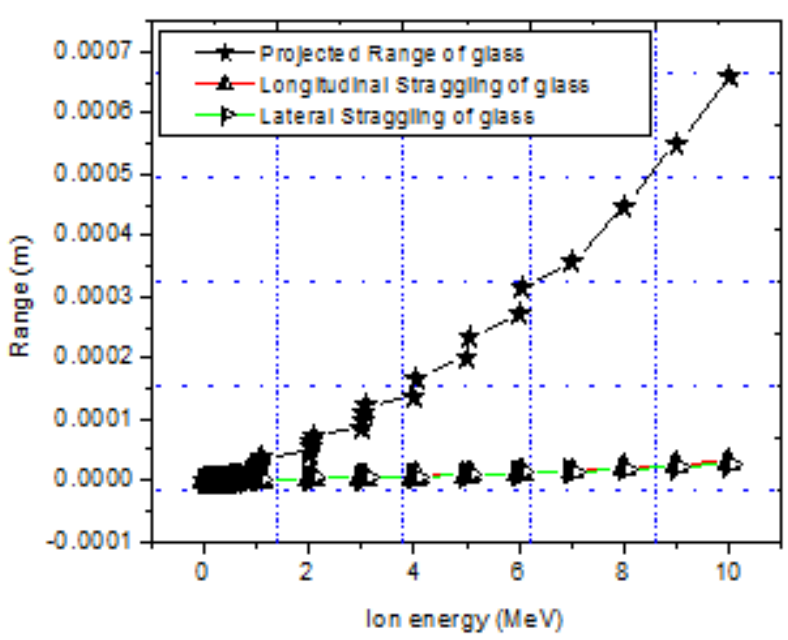

Figure 4: The range of glass for protons, plotted versus ion energies

Electronic stopping powers of $(0.01-10 \mathrm{MeV})$ protons in $\mathrm{ZnO}$ on glass target were calculated using SRIM to be (0.03661-0.5423MeV/gmcm $\left.{ }^{2}\right)$, while nuclear stopping power was $\left(0.00001819-0.004673 \mathrm{MeV} / \mathrm{gm} \mathrm{cm}^{2}\right)$. Figure 5

Volume 5 Issue 7, July 2016 www.ijsr.net 


\section{International Journal of Science and Research (IJSR) \\ ISSN (Online): 2319-7064 \\ Index Copernicus Value (2013): 6.14 | Impact Factor (2015): 6.391}

depicts the variation of electronic and nuclear stopping power of $0.01-10 \mathrm{MeV}$ protons with ion energy.

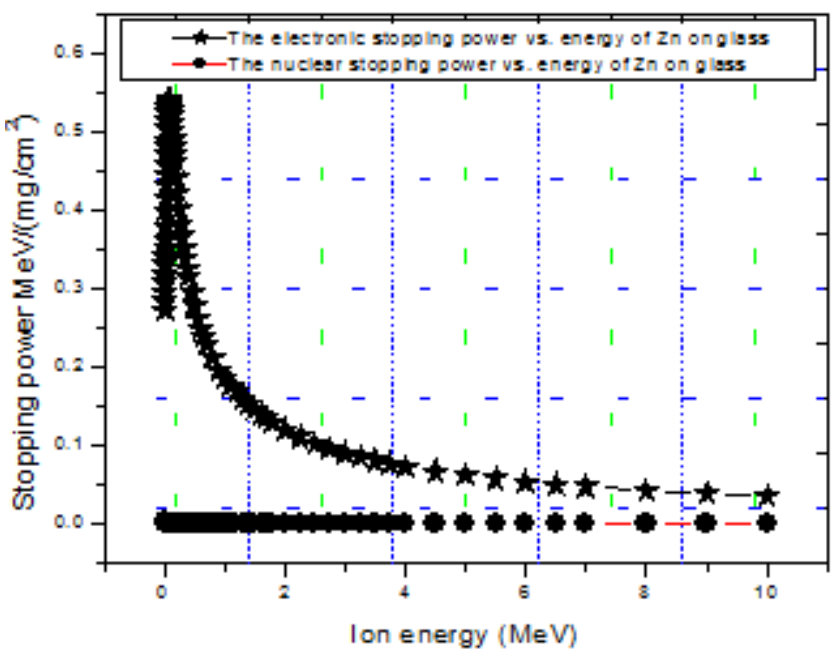

Figure 5: The stopping power of $\mathrm{ZnO}$ on glass for protons, plotted versus ion energies

The projected range, the longitudinal range and the lateral range values of $0.01-10 \mathrm{MeV}$ protons in $\mathrm{ZnO}$ on glass was calculated using SRIM to be (0.0000001514-0.00066672m), $(0.0000000571-0.00003042 \mathrm{~m})$ and $(0.00002647$ $0.0000000544 \mathrm{~m})$, respectively. Figure 6 depicts the variation of projected range, longitudinal range and lateral range of $0.01-10 \mathrm{MeV}$ protons with ion energy. The Figure 6 shows how the range of $\mathrm{ZnO}$ on glass for protons increases until it reaches the maximum. Simulation results have shown that proton beams with $10 \mathrm{MeV}$ energy experience inconsiderable broadening for the considered range of film.

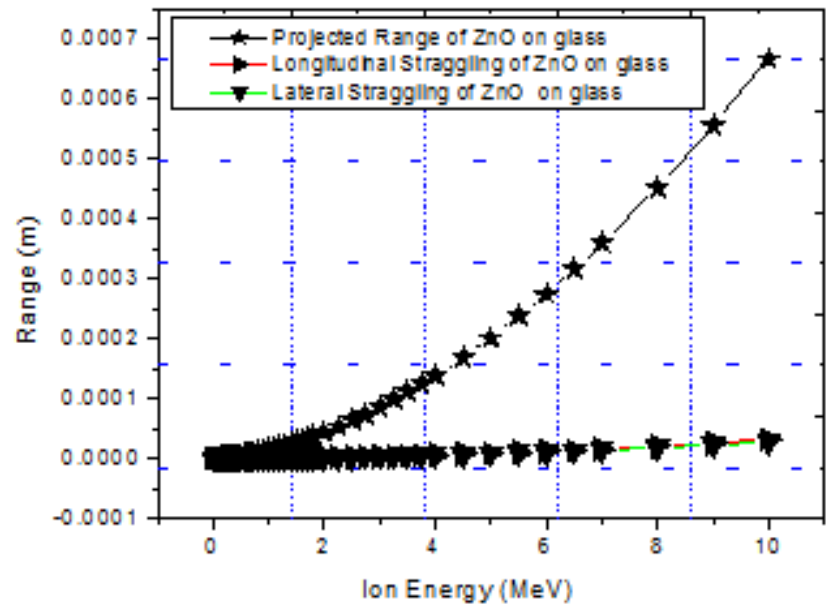

Figure 6: The range of $\mathrm{ZnO}$ on glass for protons, plotted versus ion energies

\section{Conclusion}

Simulations of proton transport through thin films of $\mathrm{ZnO}$ and glass have shown that the investigated properties may change to the passage of protons with energies higher. Nonionizing energy loss of these high energy protons is low, and they traverse the films without much atomic displacement. In the lower part of the investigated proton energy range (from $10 \mathrm{keV}$ to $1 \mathrm{MeV}$ ), however, substantial ionization losses and nonionizing energy loss are to be expected. Ionization and displacement damage produced by protons could influence the properties of these materials and compromise their reliability within complex structures.

\section{Acknowledgement}

The author wants to express his gratitude to many friends for their excellent and valuable suggestions. $\mathrm{He}$ also acknowledges help and support providing by his professors with the programs to calculate the stopping power.

\section{References}

[1] C. Bundesmann, R. Schmidt-Grund, and M. Schubert, Optical properties of $\mathrm{ZnO}$ and related compounds. In $\mathrm{K}$. Elmer, A. Klein, and B. Rech, editors, Transparent Conductive Zinc Oxide, pages Springer, (2008) 77 - 122

[2] R. Schmidt, B. Rheinl"ander and et al., Dielectric functions (1 to $5 \mathrm{eV}$ ) of wurtzite $\mathrm{MgZnO}$ thin films. Appl. Phys. Lett., 82: (2003) 2260-2262

[3] C. Klingshirn, M. Grundmann, A. Hoffmann, B.K. Meyer, and A. Waag. Zinkoxid - ein alter, neuer Halbleiter. Phys. J., 5: (2006) 33-38

[4] A. Mang, K. Reimann and St. Rübenacke, "Band Gaps, Crystal-Field Splitting, Spin-Orbit Coupling, and Exciton Binding Energies in $\mathrm{ZnO}$ under Hydrostatic Pressure," Solid State Communications, Vol. 94 , No. 4, (1995), pp 251-254. doi:10.1016/0038-1098(95)00054-2

[5] D. Kim, T. Shimomura, S. Wakaiki, T. Terashita, M. Nakayama, Physica B: Condensed Matter, 741 (2006) 376-377

[6] T. Maruyama, J. Shionoya, Journal of Materials Science Letters 11, 170 (1992)

[7] G. Srinivasan, J. Kumar, Crystal Research and Technology 41, 893 (2006)

[8] S. J. Lim, Soonju Kwon, H. Kim, Thin Solid Films 516, 1523 (2008)

[9] R. Ayouchi, D. Leinen, F. Martín, M. Gabas, E. Dalchiele, J. R. Ramos-Barrado, Thin Solid Films 426, 68 (2003)

[10] L. Zhao, J. Lian, Y. Liu, Q. Jiang, Applied Surface Science 252, 8451 (2006)

[11] M. G. Faraj, K. Ibrahim, M. H. Eisa, M. K. M. Pakhuruddin, M. Z. Pakhuruddin, Comparison of Zinc Oxide thin films deposited on the glass and polyethylene terephthalate substrates by thermal evaporation technique for applications in solar cells, OPTOELECTRONICS AND ADVANCED MATERIALS - RAPID COMMUNICATIONS Vol. 4, No. 10, ( 2010) p. 1587 1590

[12] A. N. Banerjee, C. K. Ghosh and et al., Thin Solid Films 496, 112 (2006)

[13] M. Garganourakis, S. Logothetidis and et al., Thin Solid Films 517, 6409 (2009)

[14] Yong-Seok Park, Han-Ki Kim, Soon-Wook Jeong, Woon-Jo Cho, Thin Solid Films 518, 3071 (2010)

[15] J. F. Ziegler, J. P. Biersack, and M. D. Ziegler, Stopping and Range of Ions in Matter, SRIM Co., Chester, MD (2008)

[16] T. Coffey, S. G. Urquhart and H. Ade, Characterization of the effects of soft X-ray irradiation on polymers, Journal of Electron Spectroscopy and Related Phenomena 122 (2002) 65-78 